\title{
Validation of ITS from GenBank database in diagnosing medically important black yeasts and relatives species of Herpotrichiellaceae
}

\author{
K. Samerpitak ${ }^{1^{*}}$, A.H.G. Gerrits van den Ende ${ }^{2}$ and G.S. de Hoog ${ }^{2}$ \\ ${ }^{1}$ Departmant of Microbiology, Faculty of Medicine, Khon Kaen University, Khon Kaen, Thailand \\ ${ }^{2}$ Westerdijk Fungal Biodiversity Institute, Utrecht, the Netherlands \\ * Presenting author
}

\section{Objectives}

To investigate the use of ITS from GenBank database and NCBI blast searching in diagnosing black yeast and relatives species of

Herpotrichiellaceae.

\section{Materials and Methods}

1. 356 ITS-sequences (ITS1-5.8S-ITS2) of 115 species of medical and saprophytic black yeasts and relatives in family Herpotrichiellaceae viz.Cladophialophora (33 sp.), Exophiala (40 sp.), Fonsecaea (9 sp.), Phialophora (23 sp.), Rhinocladiella (10 sp.), were collected from GenBank database by using the criteria of legitimate names in Mycobank deposition and type or the reference strains.

2. To investigate the species cluster produced from the ITS dataset, the Neighbor joining $(\mathrm{NJ})$ analysis with $\mathrm{K} 2+\mathrm{G}$ model was performed in MEGA6.

3. To observe precision and accuracy of ITS in identification, each of type or reference sequence was tested by NCBI BLAST ${ }^{\circledR}$ megablast searching for the identity values (\%).

\section{Results (Fig 1)}

1. NJ analysis;

1.1 Species boundaries of 104 species $(90.43 \%)$ were well defined with high supports.

1.2 Species boundaries of 11 species were ambiguous viz.

- 6 species, the members mixed with another species in the same cluster viz. C. devriesii \& F. brasiliensis, F. compacta \& F. pedrosoi, $P$. chinensis \& $P$. verrucosa.

- the members of 3 species, $P$. mustea, $P$. calyciformis and $P$. brunnescens were clustered together.

- the taxonomic positions of P. intermedia and P. phaeophora could not be indicated because each species member showed two different positions in the analysis.

2. NCBI megablast searching;

2.1109 species $(94.78 \%)$ were $94-100 \%$ identical with maximum scores and specific to their own types or reference species.

2.2 ITSs of six species were cross-specific to another type species viz. C. devriesii \& F. brasiliensis, F. compacta \& F. pedrosoi, $P$. chinensis \& $P$. verrucosa, concordant to the results from $\mathrm{NJ}$.

2.3 P. mustea, P. calyciformis and P. brunnescens were $99-100 \%$ identical to Pleurostoma richardsiae, the ex-member of Phialophora which is now classified into Calosphaeriaceae.

\section{Conclusion}

1. ITS sequence (ITS1-5.8-ITS2) could delimit 104 species-boundaries of black yeasts and relatives in Herpotrichiellaceae.

2. The ITS could be used as a tool to identify 109 species and highly specific to type and reference strains without cross-specific to other species (94-100\% identity).

3. Cross-specific identity might reflect ambiguous classification of some taxa, revision of them might have to be considered.

4. The hazardous causative agents of CNS infection viz. C. bantiana, $E$. dermatitidis and $R$. mackenziei, could be specifically identified by ITS with megablast searching (96-100\% identity).

5. GenBank database and NCBI blast searching are reliable and standardized enough to use for correct identification of black yeasts and relatives in Herpotrichiellaceae.

6. Validation of ITS for identifying black yeasts and relatives in other families in the GenBank database should be further investigated.
Fig 1 Neighbor joining tree from 356 ITS-sequences of 115 species of black yeasts and relatives in Herpotrichiellaceae. Numbers on the branches are percents of 1,000 bootstrap replications.

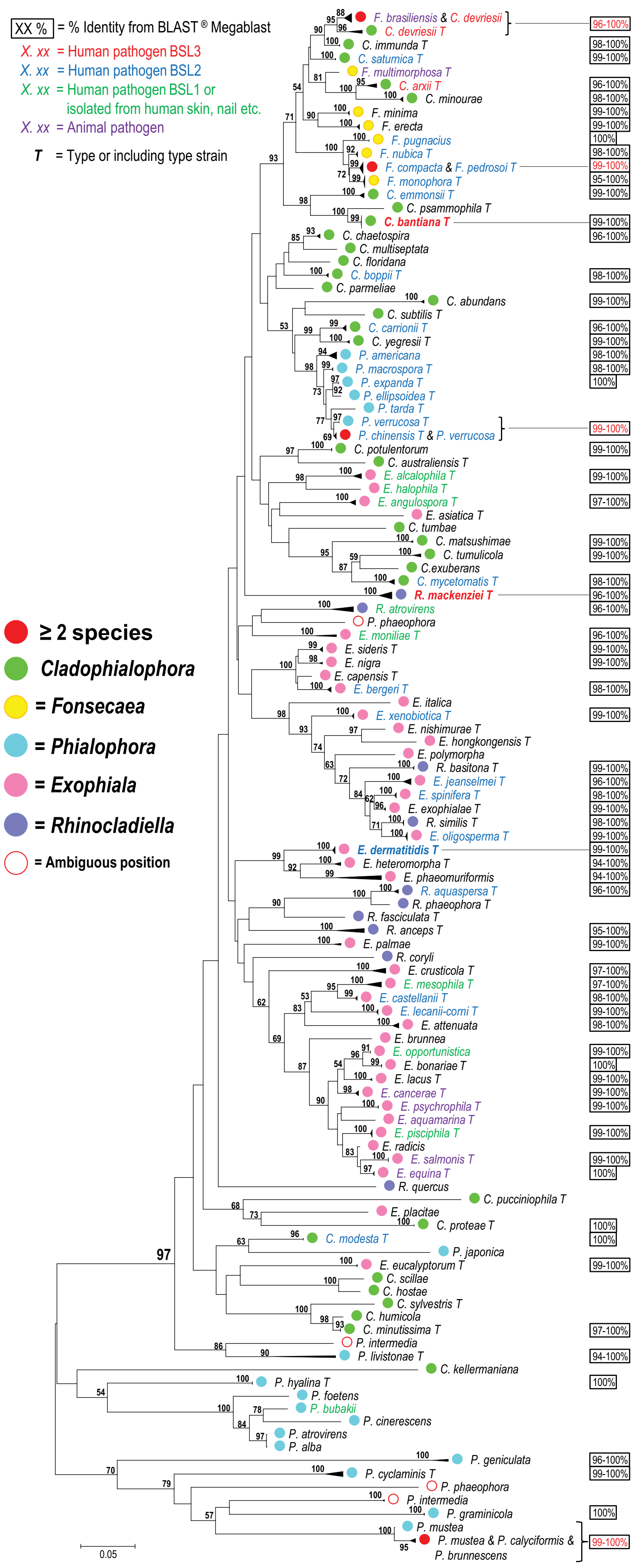

